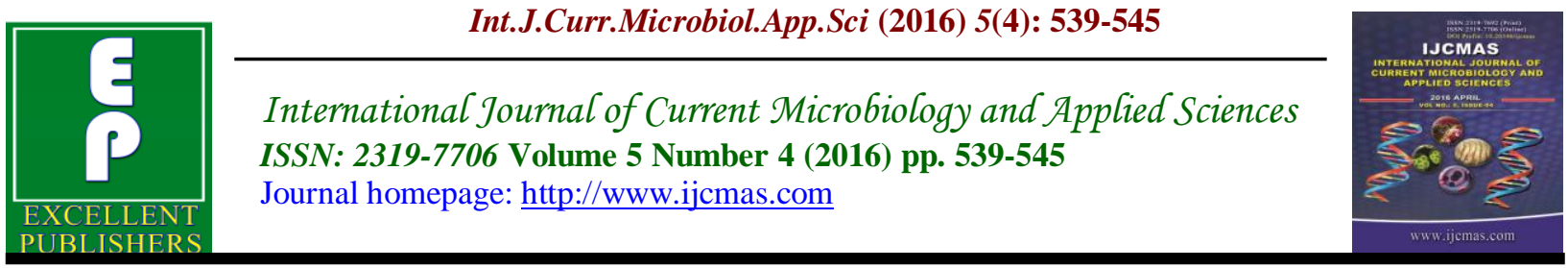

Original Research Article

http://dx.doi.org/10.20546/ijcmas.2016.504.061

\title{
A Study on Entomo-Fauna as Recorded from Cauliflower Crop in an Agro-Ecosystem near Bikaner, Rajasthan, India
}

\author{
Dheeraj Bhati and Meera Srivastava* \\ Laboratory of Entomology, Govt. Dungar College, Bikaner 334001, Rajasthan, India \\ *Corresponding author
}

\section{A B S T R A C T}

\section{Keywords}

Cauliflower, Entomo-Fauna, Agro-Ecosystem, Cage Net, Rajasthan.

\section{Article Info}

Accepted:

20 March 2016

Available Online:

10 April 2016
Cauliflower, Brassica oleracea var. botrytis L. belonging to family Brassicaceae (Cruciferae) is a very popular winter vegetable Rabi crop cultivated especially in North India. The crop is also cultivated in the State of Rajasthan during winter. Ecologically, insects play many different roles as pests, predators and parasites, pollinators, decomposers and scavengers and so forth. Looking into this, the present study was conducted to document the entomo-fauna associated with this crop in the region. An indigenously designed cage net was employed for collection. In all, 71 insects belonging to 6 orders and 32 families were collected from the crop, of which based on density 6 were dominant, 53 frequent and 12 were rare forms. The maximum density as well as diversity was found in the month of November, while, minimum in the month of April 2013.

\section{Introduction}

Cauliflower, Brassica oleracea var. botrytis L. belonging to family Brassicaceae (Cruciferae) is a very popular winter vegetable Rabi crop cultivated especially in North India. Typically, only the head (the white curd) of aborted floral meristem is eaten, while the stalk and surrounding thick, green leaves are used in vegetable broth or discarded. Cauliflower is low in fat, but high in dietary fibre, folate, water, and vitamin C, possessing a high nutritional density. Cauliflower contains several phytochemicals, usually occurring in the cabbage family that may be beneficial to human health. A high intake of cauliflower has been associated with reduced risk of aggressive prostate cancer.
The crop is also cultivated in the State of Rajasthan during winter. The state of Rajasthan, the land with vivid topographical features and geographically, is located between $23^{\circ} 3^{\prime}-30^{\circ} 12^{\prime} \mathrm{N}$ and $69^{\circ} 30^{\prime}-78^{\circ} 17^{\prime}$ E. Bikaner district lies in North-Western part of Rajasthan located between $27^{\circ} 11^{\prime}$ and $29^{\circ} 03^{\prime}$ North latitudes and $71^{\circ} 52^{\prime}$ and $74^{\circ} 12^{\prime}$ East longitudes. The district has a dry climate with large variation in temperature and has scanty rainfall. The summer months are extremely hot with the day temperature sometimes going up to $49.9^{\circ} \mathrm{C}$, May being the hottest month. During winter the minimum temperature sometimes drops up to $0^{\circ} \mathrm{C}$, January being the coldest month. Ecologically, insects play many different 
roles as pests, predators and parasites, pollinators, decomposers and scavengers and so forth. Looking into this, the present study was conducted to document the insects associated with this crop in the region.

\section{Materials and Methods}

The agro-ecosystems in the form of crop fields studied lie about 10 to $15 \mathrm{~km}$ away from the city, covering an area of 6 hectares each. These are irrigated by sewage water. In all six crop fields were covered during the present survey from September 2012 to April 2013 and September 2013 to April 2014 when the crop of cauliflower was cultivated.

An indigenously designed cage (net) of $1 \mathrm{~m} \times 1 \mathrm{~m} \times 1 \mathrm{~m}$ of nylon mesh was used for the purpose as also used by Saigal (2002). The cage covered the $1 \mathrm{~m}^{3}$ volume while holding the crop inside. The fauna trapped within the cage was mechanically picked up. Using cage the insects were collected between 7A.M to 11A.M. Sampling was done fortnightly. The insects collected by the above method were transferred to killing bottles and the killed insects were preserved. Large winged insects were put to dry preservation by pinning them in insect boxes, while, smaller insects were preserved in $70 \%$ alcohol. The fauna were sorted out group-wise and identifications were made following pertinent literature. Help from the section of Entomology, Department of Agriculture, Bikaner and Desert Regional Station of the Zoological Survey of India, Jodhpur was also taken for identification and for confirmation.

\section{Results and Discussion}

The cauliflower was cultivated in the agroecosystem during September to April. The entomofauna collected from this crop has been presented in Table 1. In all, 71 insects belonging to 6 orders and 32 families were collected from the crop, of which based on density 6 were dominant, 53 frequent and 12 were rare forms. The maximum density as well as diversity was found in the month of November, while, minimum in the month of April 2013.

Of the seventeen lepidopteran species documented, based on density, thirteen were frequent (D. chrysippus, L. boeticus, Zizina sp., E. hecabe, A. aurota, C. pomona, $C$. vestalis, $C$. indica, $H$. recurvalis, Tephrina sp., U. pulchella, S. exigua and A. ipsilon) and four (H. ornata, L. orbonalis, Hymenia sp. and $A$. styx) were rare forms. Among, twelve coleopteran species documented, eight were frequent (Cicindella sp., $A$. bengalensis, $O$. catta, $O$. bonasus, $P$. nasutus, A. ferruginea, C. septempunctata and $M$. sexmaculatus), four were (unidentified species A \& B, H. truncatulus and Myllocerus sp.) rare species. $X$. fenestrata, A. cerana and A. mellifera were documented as three dominant hymenopteran species, while, Enicospilus sp., Campsomeris sp., Scoliasoror sp., $D$. affinis, Formica sp., Pepsis sp., P. carolina, Halictus sp., $X$. violacea, A. dorsata, A. florea, unidentified species A, B and C were fourteen frequent forms.

Of the total eleven hemipteran species, eight (D. cingulatus, Clavigrella sp., N. viridula, A. janus, A. spinidens, Piezodorus sp., Oncocephalus sp. and unidentified species B) were frequent, three (B. hilaris, Alcaeus $s p$. and unidentified species C) were rare forms. Chrotogonus sp., S. gregaria, Ochrilidia sp., O. chinensis, Pyrgomorpha sp., Atractomorpha sp. and unidentified species A, were the seven frequently noted, while, only one species Acrida sp. was a rare form among the eight orthopteran insects. Among six dipteran insect species, three were dominant which included $C$. quinquefasciatus, $S$. peregrina and $M$. 
domestica and three were frequent forms (Stichopogon sp., syrphid fly and $D$. cucurbitae).

The present work gets support from the earlier work by various authors. Cruciferous vegetables viz. cabbage, cauliflower and knol-knol grown in and around Shillong have been found to be attacked by different insect pests which include cabbage butterfly Pieris brassicae, cabbage aphid Brevicoryne brassicae, mustard aphid Lipaphis erysimi, cutworms Agrotis ipsilon and A. flammatra, cabbage loopers Plusia orichalcea and Trichoplusia sp. and diamond back moth Plutella xylostella by Sachan \& Gangwar (1990). Brassica oleracea var. capitata L. is extensively grown both in hills and valleys of Manipur and is damaged by a variety of insect pests of which cabbage butterfly Pieris brassicae L. was observed as a problem pest by Shri Ram \& Pathak (1992). Mitra \& Banerjee (2007) reported cauliflower to be pollinated by flies.

Table.1 Entomofaunal Diversity and Density (Number/Trap*) on Cauliflower During the Period of Study

\begin{tabular}{|c|c|c|c|c|c|c|c|c|c|c|c|c|c|c|c|c|c|c|}
\hline & Jan & Feb & Mar & Apr & May & Jun & Jul & Aug & Sep & Oct & Nov & Dec & Jan & Feb & $\begin{array}{c}\mathbf{M a} \\
\mathbf{r}\end{array}$ & $\underset{\mathbf{r}}{\mathbf{A p}}$ & May & Status \\
\hline \multicolumn{19}{|l|}{ Order: Leptdoptera } \\
\hline \multicolumn{19}{|l|}{ Family:Danaidae } \\
\hline $\begin{array}{l}\text { Danaus chrysippus } \\
\text { Linn. }\end{array}$ & - & - & - & - & - & - & - & - & - & 2 & 3 & 3 & - & - & - & - & - & $\mathrm{F}$ \\
\hline \multicolumn{19}{|l|}{ Family:Lycaenidae } \\
\hline $\begin{array}{l}\text { Lampides boeticus } \\
\text { Linn. }\end{array}$ & - & - & - & - & - & - & - & - & - & - & 2 & 2 & 1 & - & - & - & - & $\mathrm{F}$ \\
\hline Zizina sp. & - & - & - & - & - & - & - & - & - & - & 2 & 2 & - & 1 & - & - & - & $\mathrm{F}$ \\
\hline \multicolumn{19}{|l|}{ Family:Pieridae } \\
\hline Eurema hecabe Linn. & 1 & 1 & 1 & 1 & - & - & - & - & 1 & 1 & 4 & 4 & 2 & 1 & - & 1 & - & $\mathrm{F}$ \\
\hline Anaphaeis aurota Fab. & 1 & - & - & - & - & - & - & - & 1 & 1 & 5 & 4 & 1 & - & - & - & - & $\mathrm{F}$ \\
\hline $\begin{array}{l}\text { Catopsila pomona } \\
\text { Cramer }\end{array}$ & - & 1 & 1 & & - & - & - & - & 1 & 1 & 4 & 4 & - & - & - & - & - & $\mathrm{F}$ \\
\hline Colotis vestalis Butler & - & - & 1 & 1 & - & - & - & - & - & - & 2 & 1 & - & - & - & - & - & $\mathrm{F}$ \\
\hline \multicolumn{19}{|l|}{ Family: Hesperiidae } \\
\hline $\begin{array}{l}\text { Hesperilla ornata } \\
\text { Leach. }\end{array}$ & - & 1 & - & - & - & - & - & - & - & - & - & - & 1 & - & - & - & - & $\mathrm{R}$ \\
\hline \multicolumn{19}{|l|}{ Family: Crambidae } \\
\hline $\begin{array}{l}\text { Leucinodes orbonalis } \\
\text { Guenee }\end{array}$ & - & - & - & - & - & - & - & - & - & - & 1 & 1 & - & - & - & - & - & $\mathrm{R}$ \\
\hline $\begin{array}{l}\text { Cryptographis indica } \\
\text { Saunders }\end{array}$ & - & - & - & - & - & - & - & - & - & - & 1 & 1 & 1 & 1 & - & - & - & $\mathrm{F}$ \\
\hline $\begin{array}{l}\text { Hymenia recurvalis } \\
\text { Fab. }\end{array}$ & - & - & - & - & - & - & - & - & - & - & 2 & 1 & - & 1 & - & - & - & $\mathrm{F}$ \\
\hline Hymenia sp. & - & - & - & - & - & - & - & - & - & - & 2 & - & - & - & - & - & - & $\mathrm{R}$ \\
\hline \multicolumn{19}{|l|}{ Family: Geometridae } \\
\hline Tephrina sp. & - & - & - & - & - & - & - & - & - & 1 & 2 & 1 & 1 & - & - & - & - & $\mathrm{F}$ \\
\hline \multicolumn{19}{|l|}{ Family: Sphingidae } \\
\hline Acherontia styx & - & - & - & - & - & - & - & - & - & - & - & 1 & - & - & - & - & - & $\mathrm{R}$ \\
\hline \multicolumn{19}{|l|}{ Family: Arctidae } \\
\hline $\begin{array}{l}\text { Utethesia pulchella } \\
\text { Linn. }\end{array}$ & - & - & - & - & - & - & - & - & - & - & 3 & 2 & 2 & 1 & 1 & 1 & - & $\mathrm{F}$ \\
\hline \multicolumn{19}{|l|}{ Family: Noctuidae } \\
\hline $\begin{array}{l}\text { Spodoptera exigua } \\
\text { Hubner }\end{array}$ & - & - & - & - & - & - & - & - & - & 2 & 2 & 2 & 2 & - & - & - & - & $\mathrm{F}$ \\
\hline $\begin{array}{l}\text { Agrotis ipsilon } \\
\text { Hufnagel }\end{array}$ & 1 & 1 & - & - & - & - & - & - & - & - & 3 & 2 & 1 & - & - & - & - & $\mathrm{F}$ \\
\hline \multicolumn{19}{|l|}{ Order: Coleoptera } \\
\hline \multicolumn{19}{|l|}{ Family:Cicindelidae } \\
\hline Cicindella sp. & 3 & - & - & & - & - & - & - & 1 & 1 & - & - & - & - & - & - & - & $\mathrm{F}$ \\
\hline
\end{tabular}




\begin{tabular}{|c|c|c|c|c|c|c|c|c|c|c|c|c|c|c|c|c|c|c|}
\hline & Jan & Feb & Mar & Apr & May & Jun & Jul & Aug & Sep & Oct & Nov & Dec & Jan & Feb & $\begin{array}{c}\mathrm{Ma} \\
\mathbf{r}\end{array}$ & $\begin{array}{c}\mathbf{A p} \\
\mathbf{r}\end{array}$ & May & Status \\
\hline \multicolumn{19}{|l|}{ Family: Carabidae } \\
\hline Unidentified sp. A & - & - & - & 1 & - & - & - & - & - & - & 1 & 1 & - & - & 1 & 1 & - & $\mathrm{R}$ \\
\hline \multicolumn{19}{|l|}{ Family: Scarabaeidae } \\
\hline $\begin{array}{l}\text { Anomala bengalensis } \\
\text { Blanch. }\end{array}$ & 1 & 1 & 1 & 1 & - & - & - & - & - & - & 2 & 1 & 1 & - & - & - & - & $\mathrm{F}$ \\
\hline $\begin{array}{l}\text { Onthophagus catta } \\
\text { Fab. }\end{array}$ & 2 & 2 & 1 & 1 & - & - & - & - & - & - & 2 & 3 & - & - & - & - & - & $\mathrm{F}$ \\
\hline $\begin{array}{l}\text { Onthophagus bonasus } \\
\text { Fab. }\end{array}$ & - & - & 1 & 1 & - & - & - & - & 2 & 1 & - & - & 1 & - & - & - & - & $\mathrm{F}$ \\
\hline $\begin{array}{l}\text { Peltonotus nasutus } \\
\text { Arrow }\end{array}$ & - & - & - & - & - & - & - & - & - & - & - & 2 & 2 & 2 & - & - & - & $\mathrm{F}$ \\
\hline $\begin{array}{l}\text { Apogonia ferruginea } \\
\text { Fab. }\end{array}$ & - & - & - & 2 & - & - & - & - & - & - & 2 & 1 & 3 & - & - & - & - & $\mathrm{F}$ \\
\hline Unidentified sp. B & 2 & - & - & - & - & - & - & - & - & - & - & - & 2 & - & - & - & - & $\mathrm{R}$ \\
\hline \multicolumn{19}{|l|}{ Family: Coccinellidae } \\
\hline $\begin{array}{l}\text { Coccinella } \\
\text { septempunctata } \text { Lin. }\end{array}$ & - & - & - & 4 & - & - & - & - & - & - & 2 & 2 & - & 1 & 1 & 1 & - & $\mathrm{F}$ \\
\hline $\begin{array}{l}\text { Menochilus } \\
\text { sexmaculatus Fab. }\end{array}$ & - & - & 2 & 1 & - & - & - & - & - & 2 & - & - & - & - & 2 & - & - & $\mathrm{F}$ \\
\hline \multicolumn{19}{|l|}{ Family:Curculionidae } \\
\hline $\begin{array}{l}\text { Hypolixus truncatulus } \\
\text { Fab. }\end{array}$ & - & - & - & - & - & - & - & - & - & 1 & 1 & - & - & - & 2 & - & - & $\mathrm{R}$ \\
\hline Myllocerus sp. & 1 & - & - & - & - & - & - & - & - & - & - & - & - & - & - & - & - & $\mathrm{R}$ \\
\hline \multicolumn{19}{|l|}{ Order: Hymenoptera } \\
\hline \multicolumn{19}{|l|}{$\begin{array}{l}\text { Family: } \\
\text { Ichneumonidae }\end{array}$} \\
\hline Enicospilus sp. & - & - & - & - & - & - & - & - & - & - & 3 & 2 & - & - & - & - & - & $\mathrm{F}$ \\
\hline \multicolumn{19}{|l|}{ Family:Scoliidae } \\
\hline Campsomeris sp. & - & - & - & - & - & - & - & - & - & - & 2 & 2 & - & - & - & - & - & $\mathrm{F}$ \\
\hline Scoliasoror sp. & - & - & - & - & - & - & - & - & - & 2 & 2 & 2 & - & - & - & - & - & $\mathrm{F}$ \\
\hline \multicolumn{19}{|l|}{ Family: Formicidae } \\
\hline $\begin{array}{l}\text { Dolichoderus affinis } \\
\text { Emery }\end{array}$ & - & - & - & - & - & - & - & - & - & - & 4 & 2 & 2 & - & - & - & - & $\mathrm{F}$ \\
\hline Formica sp. & - & - & - & - & - & - & - & - & - & - & 3 & 3 & - & - & - & - & - & $\mathrm{F}$ \\
\hline \multicolumn{19}{|l|}{ Family: Pompilidae } \\
\hline Pepsis sp. & - & - & - & - & - & - & - & - & - & - & 5 & 2 & - & - & - & - & - & $\mathrm{F}$ \\
\hline \multicolumn{19}{|l|}{ Family:Vespidae } \\
\hline Polistes carolina & - & - & - & - & - & - & - & - & - & 1 & 3 & 1 & - & - & - & - & - & $\mathrm{F}$ \\
\hline \multicolumn{19}{|l|}{ Family: Halictidae } \\
\hline Halictus sp. & - & - & - & - & - & - & - & - & - & 2 & 3 & 1 & 2 & - & - & - & - & $\mathrm{F}$ \\
\hline \multicolumn{19}{|l|}{ Family:Apidae } \\
\hline $\begin{array}{l}\text { Xylocopa fenestrata } \\
\text { Fab. }\end{array}$ & - & - & - & - & - & - & - & - & - & 4 & 4 & 2 & 4 & - & - & - & - & D \\
\hline $\begin{array}{l}\text { Xylocopa violacea } \\
\text { Linn. }\end{array}$ & - & - & - & - & - & - & - & - & - & 1 & 2 & 1 & 1 & - & - & - & - & $\mathrm{F}$ \\
\hline Apis cerana Fab. & - & - & - & - & - & - & - & - & - & - & 2 & 2 & - & - & - & - & - & $\mathrm{D}$ \\
\hline Apis mellifera Linn. & - & - & - & - & - & - & - & - & - & - & 5 & 5 & 4 & 2 & - & - & - & $\mathrm{D}$ \\
\hline Apis dorsata Fab. & - & - & - & - & - & - & - & - & - & 4 & 3 & 5 & - & - & - & - & - & $\mathrm{F}$ \\
\hline Apis florea Fab. & - & - & - & - & - & - & - & - & - & - & 5 & 5 & - & - & - & - & - & $\mathrm{F}$ \\
\hline Unidentified sp. A & - & - & - & - & - & - & - & - & - & 2 & 2 & 2 & 2 & - & - & - & - & $\mathrm{F}$ \\
\hline Unidentified sp. B & - & - & - & - & - & - & - & - & - & 1 & 2 & 2 & - & - & - & - & - & $\mathrm{F}$ \\
\hline Unidentified sp. C & - & - & - & - & - & - & - & - & - & - & 4 & 2 & 1 & - & - & - & - & $\mathrm{F}$ \\
\hline \multicolumn{19}{|l|}{ Order: Hemiptera } \\
\hline \multicolumn{19}{|l|}{$\begin{array}{l}\text { Family: } \\
\text { Pyrrhocoridae }\end{array}$} \\
\hline $\begin{array}{l}\text { Dysdercus cingulatus } \\
\text { Fab. }\end{array}$ & 1 & 1 & 1 & 1 & - & - & - & - & - & - & 1 & 1 & 1 & - & - & - & - & $\mathrm{F}$ \\
\hline \multicolumn{19}{|l|}{ Family:Coreidae } \\
\hline Clavigrella sp. & 3 & 2 & - & - & - & - & - & - & - & - & - & - & 3 & 1 & - & - & - & $\mathrm{F}$ \\
\hline Family: Pentatomidae & & & & & & & & & & & & & & & & & & \\
\hline Nezara viridula Linn. & 2 & 3 & - & - & - & - & - & - & 1 & 1 & - & - & - & 1 & 1 & - & - & $\mathrm{F}$ \\
\hline $\begin{array}{l}\text { Bagrada hilaris } \\
\text { Burmeister }\end{array}$ & 2 & 1 & 2 & - & - & - & - & - & 1 & - & - & - & - & - & - & - & - & $\mathrm{R}$ \\
\hline
\end{tabular}




\begin{tabular}{|c|c|c|c|c|c|c|c|c|c|c|c|c|c|c|c|c|c|c|}
\hline & Jan & Feb & Mar & Apr & May & Jun & Jul & Aug & Sep & Oct & Nov & Dec & Jan & Feb & $\begin{array}{c}\mathrm{Ma} \\
\mathbf{r}\end{array}$ & $\begin{array}{c}\mathbf{A p} \\
\mathbf{r}\end{array}$ & May & Status \\
\hline Alcaeus sp. & - & - & - & - & - & - & - & - & - & - & - & - & - & 2 & - & - & - & $\mathrm{R}$ \\
\hline Aspongopus janus Fab. & - & 1 & 1 & - & - & - & - & - & - & - & - & - & - & - & - & - & - & $\mathrm{F}$ \\
\hline $\begin{array}{l}\text { Andrallus spinidens } \\
\text { Fab. }\end{array}$ & - & - & - & - & - & - & - & - & 2 & - & - & - & - & - & - & - & - & $\mathrm{F}$ \\
\hline Piezodorus sp. & 2 & - & 2 & 2 & - & - & - & - & 2 & 2 & - & - & - & - & - & - & - & $\mathrm{F}$ \\
\hline Oncocephalus sp. & - & - & - & - & - & - & - & - & 1 & 1 & 1 & - & - & - & - & - & - & $\mathrm{F}$ \\
\hline Unidentified sp. B & - & - & 2 & 3 & - & - & - & - & 2 & 2 & - & - & - & - & - & - & - & $\mathrm{F}$ \\
\hline Unidentified sp. C & - & - & - & - & - & - & - & - & 1 & 1 & 1 & - & - & - & - & - & - & $\mathrm{R}$ \\
\hline \multicolumn{19}{|l|}{$\begin{array}{l}\text { Order: } \\
\text { Orthoptera }\end{array}$} \\
\hline \multicolumn{19}{|l|}{ Family:Acrididae } \\
\hline Chrotogonus sp. & 1 & 1 & 1 & - & - & - & - & - & 1 & 1 & - & - & - & - & - & - & - & $\mathrm{F}$ \\
\hline $\begin{array}{l}\text { Schistocerca } \\
\text { gregaria Forskal }\end{array}$ & 2 & 1 & 1 & 1 & - & - & - & - & - & - & 1 & 1 & 1 & - & - & - & - & $\mathrm{F}$ \\
\hline Ochrilidia sp. & - & 1 & 1 & 1 & - & - & - & - & 1 & 1 & 1 & - & - & - & - & - & - & $\mathrm{F}$ \\
\hline $\begin{array}{l}\text { Oxya chinensis } \\
\text { Thunberg }\end{array}$ & - & - & 1 & 1 & - & - & - & - & 1 & 1 & 1 & 1 & 1 & 1 & 1 & - & - & $\mathrm{F}$ \\
\hline Acrida sp. Linn. & - & - & 2 & - & - & - & - & - & - & - & - & - & - & - & - & - & - & $\mathrm{R}$ \\
\hline \multicolumn{19}{|l|}{$\begin{array}{l}\text { Family: } \\
\text { Pyrgomorphidae }\end{array}$} \\
\hline Pyrgomorpha sp. & 2 & - & 2 & 2 & - & - & - & - & 2 & 1 & - & - & - & - & - & - & - & $\mathrm{F}$ \\
\hline Atractomorpha sp. & - & - & - & - & - & - & - & - & 1 & 1 & - & - & 2 & 2 & 2 & - & - & $\mathrm{F}$ \\
\hline Unidentified sp. A & - & - & - & - & - & - & - & - & - & - & 2 & - & - & 1 & - & 1 & - & $\mathrm{F}$ \\
\hline \multicolumn{19}{|l|}{ Order:Diptera } \\
\hline \multicolumn{19}{|l|}{ Family:Culicidae } \\
\hline $\begin{array}{l}\text { Culex } \\
\text { quinquefasciatus } \\
\text { Say }\end{array}$ & 6 & 4 & 5 & 3 & - & - & - & - & 3 & 2 & 4 & 3 & 2 & 3 & 4 & 5 & - & $\mathrm{D}$ \\
\hline \multicolumn{19}{|l|}{ Family:Asilidae } \\
\hline Stichopogon sp. & 1 & - & 1 & 1 & - & - & - & - & 2 & 2 & - & - & - & 1 & 2 & 3 & - & $\mathrm{F}$ \\
\hline \multicolumn{19}{|l|}{$\begin{array}{l}\text { Family: } \\
\text { Syrphidae }\end{array}$} \\
\hline Syrphid fly & 1 & 1 & 1 & - & - & - & - & - & 1 & - & 1 & 1 & - & - & - & - & - & $\mathrm{F}$ \\
\hline \multicolumn{19}{|l|}{$\begin{array}{l}\text { Family: } \\
\text { Tephritidae }\end{array}$} \\
\hline Dacus cucurbitae & 2 & 1 & 1 & - & - & - & - & - & - & - & - & - & 1 & 2 & 1 & 2 & - & $\mathrm{F}$ \\
\hline \multicolumn{19}{|l|}{$\begin{array}{l}\text { Family: } \\
\text { Sarcophagidae }\end{array}$} \\
\hline $\begin{array}{l}\text { Sarcophaga } \\
\text { peregrina }\end{array}$ & 2 & 1 & 1 & 1 & - & - & - & - & 2 & - & 2 & - & 1 & 1 & - & - & - & $\mathrm{D}$ \\
\hline \multicolumn{19}{|l|}{ Family:Muscidae } \\
\hline $\begin{array}{l}\text { Musca domestica } \\
\text { Fab. }\end{array}$ & 2 & 2 & 2 & 2 & - & - & - & - & 2 & 2 & 2 & 2 & 1 & 1 & 1 & 1 & - & $\mathrm{D}$ \\
\hline
\end{tabular}

Chryso dieixis sp. has been noted to attack, plants belonging to cabbage family (Swain, 1971; Holloways, 1964). Spodoptera litura has been reported to cause damage to cabbage (Hick, 1980; Hill, 1975). Pieris brassicae is one of the most destructive pests of cole crops and has been reported to cause damage in entire Himalayan ranges as well as in foot hills by Rataul (1966). Heliothis armigera damages a wide range of crops including cabbage and Agrotis ipsilon has been reported to damage cabbage (Hill, 
1975; Hick 1980). In plains of Rajasthan, cabbage aphid incidence has been reported from November to March there after declining due to heat by Sachan \& Srivastava (1972). Honeybees as effective pollinators of cauliflower were also reported by Kakar (1981). Zaz \& Kuchwaha (1983) made a relative quantitative survey of the tobacco caterpiller, Spodoptera litura on cauliflower and cabbage crops during both kharif and rabi seasons. The authors recorded the pest from last week of June till last week of December on cauliflower, which shifted subsequently to cabbage wherlin the incidence commenced from end of December or first week of January and lasted till the end of March. The pest activity on cauliflower crop got accelerated about middle of August and again in November. On cabbage its peak activity was recorded during March. Free (1993) reported flies and bees to visit cauliflower. Kumar \& Nigam (1991) have noted cauliflower to be attacked by many insects viz., Lipaphis erysimi, Myzus persicae, Brevicoryne brassicae, Euborellia annulips, Tricentrus bicolor, Patanga succinata, Bemisia tabaci, Caliothrips indicus, Frankliniella sulphurea, Thrips, Plutella maculipennis, Hellula undalis, Diacrisia obliqua, Crocidolomia binotalis, Agrotis ipsilon, Agrotis segatum, Plusia chalcites, Plusia eriosoma, $P$. orichalcea, P. signata, Trichoplusia, Spodoptera litura, Phytomyza atricornis, Athalia proxima, Chaetochnema basalis, Phyllatreata cruciferae and Apion spp. and therefore support the present findings.

Cauliflower crop has been observed to be attacked by various pests which included, Patanga succincta, Tricentrus bicolor, Lipaphis erysimi, Myzus persicae, Bagrada cruciferarum, Caliothrips idicus, Thrips tabaci, Plutella xylostella, Crocidolomia binotalis, Hellula undalis, Diacrisia obliqua, Amathes c-nigrum, Agrotis ipsilon,
A. segetum, Spodoptera litura, Trichoplusia, Phytomyza atricornis, Athalia lugens proxima and Dorylus orientalis as suggested by Nayar et al. (1998) which are also in conformation with the present study. Besides, some other works which support the present findings include those by Sima \& Srivastava (2012; 2014), Sima et al. (2014) and Bhardwaj et al. (2010, 2012 and 2014), wherein the authors have documented various insects on different crops in the desert region.

\section{References}

Bhardwaj, H., Bhati, D., Srivastava, M. 2014. Insect visitors to inflorescence of coriandrum sativum as observed in an agro-ecosystem near Bikaner, Rajasthan (India). Asian Acad. Res. J. Multidisciplinary, 1(27): 396-408.

Bhardwaj, Harshwardhan, Parul Thaker, Meera, Srivastava. 2010. Hymenopteran visitors of Tagetes erecta as observed in an agroecosystem near Bikaner, Rajasthan. Curr. Biotica, 4(1): 94-102.

Bhardwaj, Harshwardhan, Srivastava, Meera. 2012. A study on insect visitors of certain cucurbit vegetable crops in an agro-ecosystem near Bikaner, Rajasthan, India. J. Acad., 2(3): 99-126.

Bhardwaj, Harshwardhan, Thaker, Parul, Srivastava, Meera. 2012. Hymenopteran floral visitors as recorded from an agro-ecosystem near Bikaner, Rajasthan. Global J. Sci. Front. Res. Agric. Bio., 12(3): 19-34.

Free, J.B. 1993. Insect pollination of crops $\left(2^{\text {rd }}\right.$ ed.) Academic press, Harcourt Brace Jovanovich Publ., London. 684 p.

Hick, S. 1980. Insect and Nematode Pests of Crops. Rural Development Hand book No. 17 DPI. 
Hill, D.S. 1975. Agricultural Insect pests of the Tropics \& their control. Cambridge University Press, London.

Holloway, J.K. 1964. Projects in Biological control of weeds. pp. 650 - 670 in Paul De Bach, editor, Biological control of Insects Pests and weeds.

Kakar, K.L. 1981. Foraging behavior of insect pollination of Cauliflower bloom. Indian J. Ecol., 8: 126-130.

Kumar, A., Nigam, P.M. 1991. Economic and applied entomology. Emkay Publications, New Delhi. pp. 530.

Mitra, B., Banerjee, D. 2007. Fly pollinators: assessing their value in biodiversity conservation and food security in India. Rec. Zool. Surv. India, 107(1): 33-48.

Nayar, K.K., Ananthakrishnan, T.N., David, B.V. 1998. General and applied entomology. Tata McGraw-Hill Publishing Company Limited, New Delhi. pp. 589.

Rataul, H.S. 1966. Biology of cabbage butterfly, Pieris brassicae L. Indian J. Hort., 16: 256-265.

Sachan, J.N., Gangwar, S.K. 1990. Seasonal incidence of insect pests of cabbage, cauliflower and Knol-Khol. Indian J. Ent., 52(1): 11-124.

Sachan., J.N., Srivastava, B.P. 1972. Studies on the seasonal incidence of insect pests of cabbage. Indian J. Ent., 34(2): 123-129.

Saigal, D. 2002. A study on entomofauna and its ecology in some agroecosystem in the arid around Bikaner (NW Rajasthan). Ph.D Thesis, Maharshi Dayanand Saraswati University,
Ajmer. pp. 1-129.

Shri Ram, Pathak, K.A. 1992. Insecticidal control for cabbage butterfly, Pieris brassicae Linn. (Pieridae: Lepidoptera) in Manipur. Indian $J$. Ent., 54(3): 353-355.

Sima, Dheeraj Bhati, Meera Srivastava. 2014. Floral visitors of different crops as recorded from an agro-ecosystem near Jhunjhunu, Rajasthan (India). Int. J. Sci. Res., 3(9): 1732-1738.

Sima, Srivastava, Meera. 2012. Entomofauna associated with Bajra crop as observed in an agro-ecosystem in Rajasthan, India. Int. J. Theoretical \& Appl. Sci., 4(2): 109-121.

Sima, Meera Srivastava. 2014. A comparative study of insect collection made by employing two different methods of collection in an agroecosystem near Jhunjhunu, Rajasthan (India). Int. J. Sci. Res., 3(9): 17391748.

Swain, G. 1971. Foreign and commonwealth office, Overseas Development Administration, Overseas Resear Publication. Ag. Zool. in Fiji. HMSO 18, London, $424 \mathrm{pp}$.

Swami, H., Joshi, F.L., Sharma, U.S. 1998. A note on the incidence of major insect pests of cabbage, Brassica oleracea var. capita Linn. Indian J. App. Ent., 12: 71-73.

Zaz, G.M., Kushwaha, K.S. 1983. Quantitative incidence of tobacco caterpillar, Spodoptera litura (F.) and related natural enemies in cole corps. Indian J. Ent., 45(2): 201-202.

\section{How to cite this article:}

Dheeraj Bhati and Meera Srivastava. 2016. A Study on Entomo-Fauna as Recorded from Cauliflower Crop in an Agro-Ecosystem near Bikaner, Rajasthan, India. Int.J.Curr.Microbiol.App.Sci.5(4): 539-545. doi: http://dx.doi.org/10.20546/ijcmas.2016.504.061 